\title{
Seismic processing in the inverse data space
}

\author{
A. J. Berkhout ${ }^{1}$
}

\begin{abstract}
Until now, seismic processing has been carried out by applying inverse filters in the forward data space. Because the acquired data of a seismic survey is always discrete, seismic measurements in the forward data space can be arranged conveniently in a data matrix $(\mathbf{P})$. Each column in the data matrix represents one shot record. If we represent seismic data in the temporal frequency domain, then each matrix element consists of a complex-valued number. Considering the dominant role of multiple scattering in seismic data, it is proposed to replace data matrix $\mathbf{P}$ by its inverse $\mathbf{P}^{-1}$ before starting seismic processing. Making use of the feedback model for seismic data, multiple scattered energy is mapped onto the zero time axis of the inverse data space. The practical consequence of this remarkable property may be significant: multiple elimination in the inverse data space simplifies to removing data at zero time only. Moving to the inverse data space may cause a fundamental change in the way we preprocess and image seismic data.
\end{abstract}

\section{INTRODUCTION}

After the introduction of seismic digital signal processing in the 1960 s, seismic processing in the 1970 s was characterized by the time series approach. Processes such as statistical deconvolution, common-midpoint stacking, and time migration treated seismic data as time objects. In the early 1980s, however, wave theory regained a principal role. The seismic community realized that seismic data should be considered as sampled wavefields, measured with proper aliasing protection at the data acquisition surface. Transformation of surface measurements into wavefields below the acquisition surface — wavefield extrapolation - evolved into one of the key operations in seismic signal processing. In addition, wave-theory-based multiple removal algorithms were successfully introduced.

In this paper, a concept is proposed that may introduce a new tran- sition path in seismic processing. It is shown that working in the inverse data space offers new opportunities in preprocessing and imaging.

\section{ARRANGING SEISMIC MEASUREMENTS IN A FORWARD DATA MATRIX}

It has been shown (Berkhout, 1982) that measurements of a seismic survey can be arranged conveniently in a data matrix, with each column representing a shot record and each row representing a receiver gather (Figure 1). This matrix can be used directly for the formulation of wave-theory-based numerical algorithms in seismic processing, such as multiple removal and prestack migration. In many theoretical considerations, the data matrix is assumed to be completely filled with regularly sampled measurements. Figure 1 illustrates that this is not the case in practice. In particular, the spacing between submatrices may be large. Note that in the temporal frequency domain, each element of the data matrix represents the frequency component of a single seismic trace; i.e., one complex-valued number. After removal of the waves that have travelled along the surface, the data matrix can be expressed in terms of propagation and reflection operators, yielding the WRW-model (Berkhout, 1982). In this paper, the data matrix plays a central role.

\section{OPERATOR FRAMEWORK FOR WAVEFIELDS}

The detail-hiding operator framework for wavefields is an attractive starting point for the derivation of seismic processing algorithms. In the single scattering version of this framework, the discrete version of the model for primary wavefields is formulated in the $\left(\mathrm{x}_{\mathrm{r}}, \mathrm{y}_{\mathrm{r}} ; \mathrm{x}_{\mathrm{s}}, \mathrm{y}_{\mathrm{s}} ; \omega\right)$-domain in terms of vectors and matrices (Figure 2):

$$
\Delta \mathbf{P}_{\mathrm{j}}\left(\mathrm{z}_{0}, \mathrm{z}_{0}\right)=\mathbf{D}\left(\mathrm{z}_{0}\right) \Delta \mathbf{X}\left(\mathrm{z}_{0}, \mathrm{z}_{0}\right) \mathbf{S}_{j}\left(\mathrm{z}_{0}\right) .
$$

In equation 1a, detector matrix $\mathbf{D}\left(\mathrm{z}_{0}\right)$ and source vector $\mathbf{S}_{\mathrm{j}}\left(\mathrm{z}_{0}\right)$ represent the angle-dependent data acquisition information for one shot record (geometry, field arrays, and signature) at the surface $z=\mathrm{z}_{0, j}$ indicating the lateral position of the source array; matrix $\Delta \mathbf{X}\left(\mathrm{z}_{0}, \mathrm{z}_{0}\right)$ defines the earth's multidimensional transfer operator for primary

Manuscript received by the Editor December 7, 2005; revised manuscript received January 22, 2006; published online August 3, 2006

${ }^{1}$ Delft University of Technology, The Netherlands.E-mail: a.j.berkhout@ tudelft.nl.

(c) 2006 Society of Exploration Geophysicists. All rights reserved. 
reflections (each column represents one spatial impulse response). Note that $\Delta \mathbf{X}$ includes mode conversion.

For OBC-data, the detector surface $\left(\mathrm{z}_{0}\right)$ should be replaced by the sea bottom $\left(z_{1}\right)$, where $z_{1}$ may be generalized to $z_{1}(x, y)$ :

$$
\Delta \mathbf{P}_{\mathrm{j}}\left(\mathrm{z}_{1}, \mathrm{z}_{0}\right)=\mathbf{D}\left(\mathrm{z}_{1}\right) \Delta \mathbf{X}\left(\mathrm{z}_{1}, \mathrm{z}_{0}\right) \mathbf{S}_{j}\left(\mathrm{z}_{0}\right)
$$

It follows from equation 1a that one trace at detector (array) position $i$ due to a seismic source (array) at position $j$ is given by the complexvalued scalar:

$$
\Delta \mathbf{P}_{\mathrm{ij}}\left(\mathrm{z}_{0}, \mathrm{z}_{0}\right)=\mathbf{D}_{\mathrm{i}}^{\dagger}\left(\mathrm{z}_{0}\right) \Delta \mathbf{X}\left(\mathrm{z}_{0}, \mathrm{z}_{0}\right) \mathbf{S}_{\mathrm{j}}\left(\mathrm{z}_{0}\right)
$$

a) Marine situation

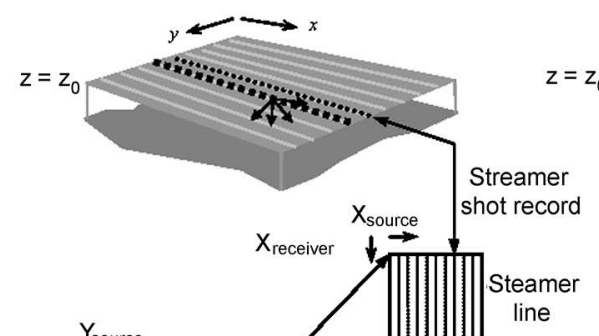

b) Land situation
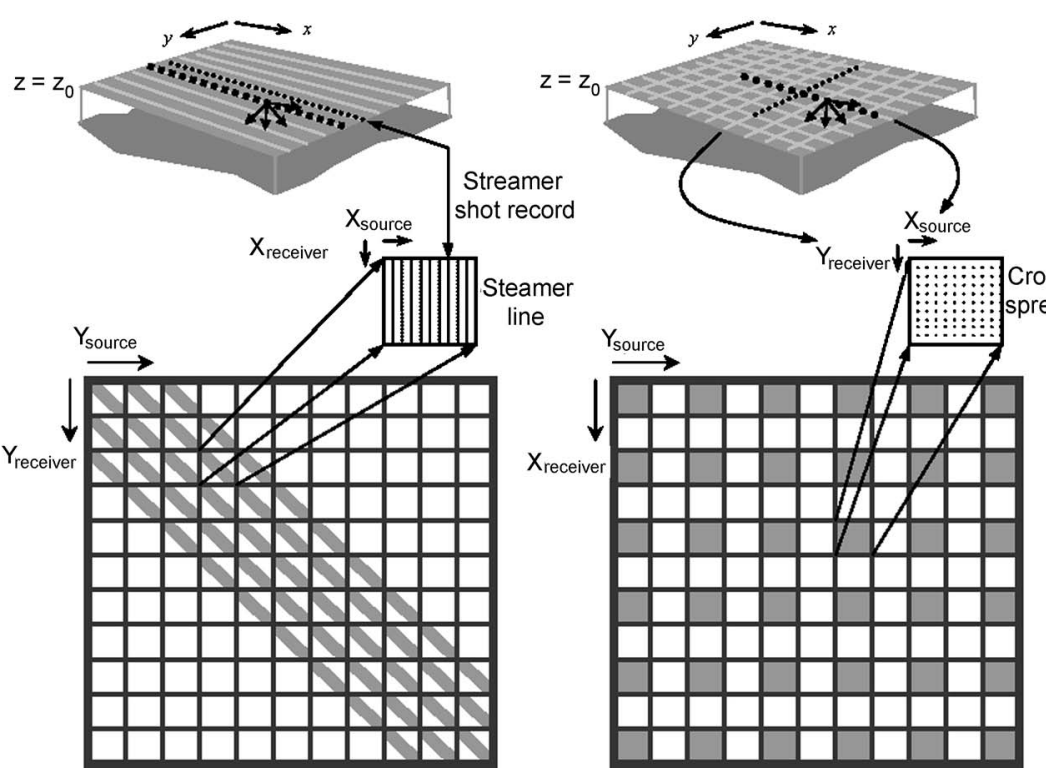

Figure 1. The data matrix for 3D seismic measurements, one column representing a shot record and one row representing a receiver gather. (a) In multistreamer marine data, one submatrix represents the measurements of a single streamer line ( 5 streamers are shown in this example). (b) In multicross-spread land data, one submatrix represents the measurements of a single cross-spread (36 cross-spreads are shown here). In practice, submatrices are generally finely sampled.

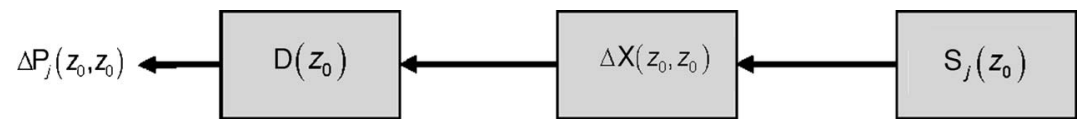

Figure 2. Primary reflection measurements in terms of surface operators $(\mathbf{S}, \mathbf{D})$ and subsurface operator $(\Delta \mathbf{X})$, where $\Delta \mathbf{X}$ includes mode conversion.

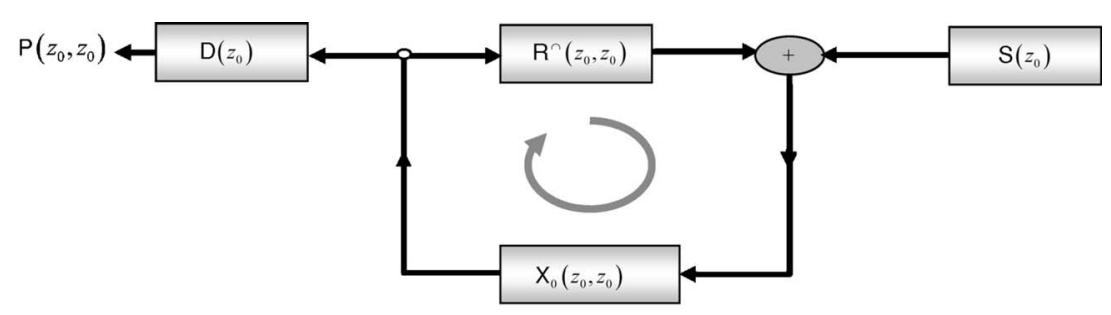

Figure 3. Feedback model, showing the generation of surface-related multiples (Berkhout, 1982). The mathematics of this model leads to a set of multidimensional integral equations of the second kind. Note that if we neglect the internal multiples, then $\mathbf{X}_{0}$ $=\Delta \mathbf{X}$. row vector $\mathbf{D}_{\mathrm{i}}^{\dagger}\left(\mathrm{z}_{0}\right)$ representing the detector (array) at position $i$. If same detector distribution (OBC data for instance), then it follows we include multiples $(\mathbf{M})$ and other types of noise $(\mathbf{N})$, such as waves that travel along the surface, the total data matrix needs to be $\mathbf{P}\left(\mathrm{z}_{0}, \mathrm{z}_{0}\right)=\Delta \mathbf{P}\left(\mathrm{z}_{0}, \mathrm{z}_{0}\right)+\mathbf{M}\left(\mathrm{z}_{0}, \mathrm{z}_{0}\right)+\mathbf{N}\left(\mathrm{z}_{0}\right)$ or

$$
\mathbf{P}\left(\mathrm{z}_{0}, \mathrm{z}_{0}\right)=\mathbf{D}\left(\mathrm{z}_{0}\right) \mathbf{X}\left(\mathrm{z}_{0}, \mathrm{z}_{0}\right) \mathbf{S}\left(\mathrm{z}_{0}\right)+\mathbf{N}\left(\mathrm{z}_{0}\right)
$$

$\mathbf{X}\left(\mathrm{z}_{0}, \mathrm{z}_{0}\right)$ being the earth's multidimensional transfer operator for all types of reflections (primaries and multiples, longitudinal and shear).

If the surface multiples have been removed, then the stress-free surface has been replaced by a reflection-free surface, and equations $3 \mathrm{a}$ and $3 \mathrm{~b}$ need to be replaced by

$$
\begin{aligned}
\mathbf{P}_{0}\left(\mathrm{z}_{0}, \mathrm{z}_{0}\right)= & \Delta \mathbf{P}\left(\mathrm{z}_{0}, \mathrm{z}_{0}\right)+\mathbf{M}_{0}\left(\mathrm{z}_{0}, \mathrm{z}_{0}\right) \\
& +\mathbf{N}\left(\mathrm{z}_{0}\right)
\end{aligned}
$$

or

$$
\mathbf{P}_{0}\left(\mathrm{z}_{0}, \mathrm{z}_{0}\right)=\mathbf{D}\left(\mathrm{z}_{0}\right) \mathbf{X}_{0}\left(\mathrm{z}_{0}, \mathrm{z}_{0}\right) \mathbf{S}\left(\mathrm{z}_{0}\right)+\mathbf{N}\left(\mathrm{z}_{0}\right) .
$$

The subscript 0 indicates that the influence of the surface $\left(\mathrm{z}_{0}\right)$ has been removed. Note that if we neglect the internal multiples, then $\mathbf{P}_{0}=\Delta \mathbf{P}$ and $\mathbf{X}_{0}=\Delta \mathbf{X}$.

In the following, we take a closer look at total data matrix $\mathbf{P}$.

\section{MAKING USE OF THE FEEDBACK MODEL}

Let us consider the feedback model at $\mathrm{z}_{0}$, showing the physics behind surface multiples (Figure 3):

$\mathbf{P}=\mathbf{P}_{0}+\left(\mathbf{P}_{0} \mathbf{A}\right) \mathbf{P}_{0}+\left(\mathbf{P}_{0} \mathbf{A}\right)^{2} \mathbf{P}_{0}+\ldots \ldots$,

where

$$
\mathbf{P}_{0}=\mathbf{D X}_{0} \mathbf{S}
$$

and

$$
\mathbf{A}=\mathbf{S}^{-1} \mathbf{R}^{\cap} \mathbf{D}^{-1} .
$$

Bear in mind that $\mathbf{P}_{0}$ contains internal multiples only.

In mathematical terms, multiplication with $\left(\mathbf{P}_{0} \mathbf{A}\right)$ means a spatial convolution process. In physical terms, multiplication with $\left(\mathbf{P}_{0} \mathbf{A}\right)$ means adding one roundtrip through the subsurface. Equation 5 a can also be written as: 


$$
\mathbf{P}=\left[\mathbf{I}-\mathbf{P}_{0} \mathbf{A}\right]^{-1} \mathbf{P}_{0}
$$

or

$$
\mathbf{P}=\mathbf{P}_{0}+\mathbf{P}_{0} \mathbf{A P} .
$$

The continuous formulation of equation $6 \mathrm{~b}$ represents a set of multidimensional integral equations of the second kind. Multiple scattering equation $6 \mathrm{~b}$ defines the theoretical base of multiple removal algorithms such as SRME (Berkhout, 1982). Note that equation $6 \mathrm{~b}$ has the structure of the well-known Lippmann-Schwinger equation (Weglein et al., 1997). For practical purposes, it is essential that equation $6 \mathrm{~b}$ includes the influence of the data acquisition operators $\mathbf{D}$ and $\mathbf{S}$ (Verschuur, 1991; Kelamis and Verschuur, 2000). It is also important to realize that surface operator $\mathbf{A}$ does not contain traveltime (see equation $5 \mathrm{c}$ ). This property of $\mathbf{A}$ is used in the next section.

\section{MOVING TO THE INVERSE DATA SPACE}

According to the feedback model (Figure 3), multiple scattering data in the forward data space (FDS) is given by

$$
\mathbf{P}=\left[\mathbf{I}-\mathbf{P}_{0} \mathbf{A}\right]^{-1} \mathbf{P}_{0} .
$$

The series expansion of equation $7 \mathrm{a}$ is given by equation 5a, showing that in practice the forward data space may be very complex. From expression $7 \mathrm{a}$, multiple scattering data in the inverse data space (IDS) can be easily derived:

or

$$
\mathbf{P}^{-1}=\mathbf{P}_{0}^{-1}\left[\mathbf{I}-\mathbf{P}_{0} \mathbf{A}\right]
$$

$$
\mathbf{P}^{-1}=\mathbf{P}_{0}^{-1}-\mathbf{A} \text {. }
$$

Equation $7 \mathrm{~b}$ may be referred to as the multiple scattering equation in the inverse data space. It shows that the inverse data space is very simple with respect to the forward data space, consisting of the inverse surface-free response, primarily situated at negative times, and the surface-related properties at and around zero time. This can be well understood if we bear in mind that the inversion process transforms the poles in the reverberant forward data to zeros in the nonreverberant inverse data. If we illustrate this with a single reflector being illuminated by a plane wave, then matrices become scalars (see Figure 4):

$$
\begin{gathered}
\mathrm{P}=\left[1+\operatorname{Re}^{-2 \mathrm{j} \omega \mathrm{p}_{\mathrm{z}} \Delta \mathrm{z}}\right]^{-1}\left[\mathrm{DRe}^{-2 \mathrm{j} \omega \mathrm{p}_{\mathrm{z}} \Delta \mathrm{z}} \mathrm{S}\right] \\
\mathrm{P}^{-1}=\left[\mathrm{DRe}^{-2 \mathrm{j} \omega \mathrm{p}_{\mathrm{z}} \Delta \mathrm{z}} \mathrm{S}\right]^{-1}+(\mathrm{DS})^{-1} \\
\mathrm{P}_{0}^{-1}=\Delta \mathrm{P}^{-1}=\left[\mathrm{DRe}^{-2 \mathrm{j} \omega \mathrm{p}_{\mathrm{z}} \Delta \mathrm{z}} \mathrm{S}\right]^{-1} \text { and } \mathrm{A}=-(\mathrm{DS})^{-1} \quad(8 \mathrm{c}) \\
\mathrm{P}_{0}=\Delta \mathrm{P}=\mathrm{DRe}^{-2 \mathrm{j} \omega \mathrm{p}_{\mathrm{z}} \Delta \mathrm{z}} \mathrm{S} \text { and } \mathrm{X}_{0}=\Delta \mathrm{X}=\operatorname{Re}^{-2 \mathrm{j} \omega \mathrm{p}_{\mathrm{z}} \Delta \mathrm{z}},
\end{gathered}
$$

where $\mathrm{p}_{\mathrm{z}} \Delta \mathrm{z}=200 \mathrm{~ms}$ and $\mathrm{R}=0.5$. Figure 4 clearly demonstrates the simplicity of $\mathrm{P}^{-1}$ with respect to $\mathrm{P}$, as predicted by the theory.

Surface operator $\mathbf{A}$ can be found at and around zero time. Knowing that the surface is stress free for marine data, meaning $\mathbf{R}^{\cap}\left(\mathrm{z}_{0}, \mathrm{z}_{0}\right)=-\mathbf{I}$, it follows from its definition that matrix $\mathbf{A}$ contains the data acquisition information (wavelet and directivity) for each c)

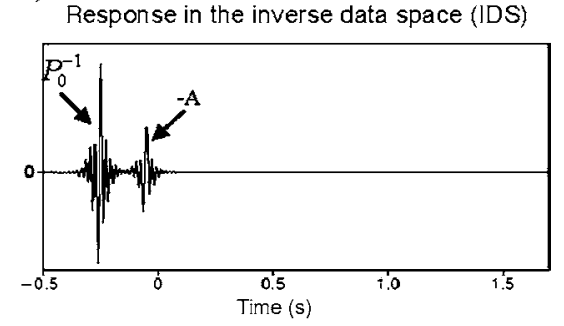

e)

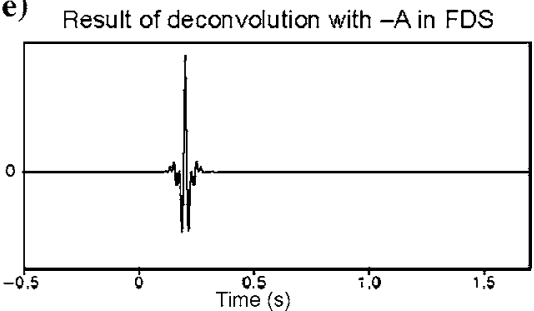

Figure 4. (a) A plane wave, single reflector example to show the difference between (b) P the forward data space and (c) $\mathrm{P}$ in the inverse data space. After removing $\mathrm{A}$ in the inverse data space, (d) $\mathrm{P}_{0}$ and (e) $\mathrm{X}_{0}=-\mathrm{AP}_{0}$ can be recovered. Note that the output is multiple free and zero phase. Note also that for this single reflector example $\mathrm{X}_{0}=\Delta \mathrm{X}$.

Response in the forward data space (FDS)
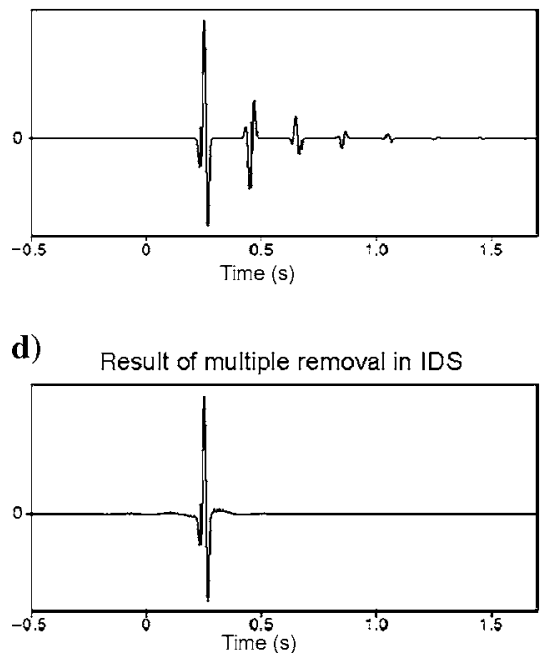

Plane wave in a single reflector model $\left(p_{z} \Delta z=200 \mathrm{~ms}\right)$

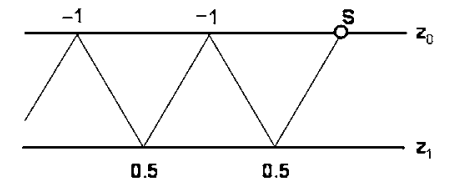


shot record as materialized in the field. Hence, A can be used to apply a full deconvolution process for sources and detectors (see Figure 5). This capability may offer new opportunities for the improvement of time-lapse preprocessing.

\section{SYNTHETIC DATA EXAMPLE}

Preprocessing in the inverse data space is demonstrated on finitedifference data in a horizontally-layered medium that was derived from log data. Figure 6a shows one shot record (primaries plus surface as well as internal multiples), and Figure 6b shows this shot record without surface multiples (primaries plus internal multiples), both in the forward data space: one column of $\mathbf{P}$ and $\mathbf{P}_{0}$ in the time domain. In Figures $6 \mathrm{c}$ and d, both records are shown in the inverse data space: one row of $\mathbf{P}^{-1}$ and $\mathbf{P}_{0}^{-1}$ in the time domain. Here, matrix inversion was carried out in the spatial Fourier domain. In Figure $6 \mathrm{c}$, the focal point at and around zero time makes the difference with respect to Figure 6d. In Figures $6 \mathrm{e}$ and $\mathrm{f}$, both shot records are shown in the Radon-transformed inverse data space. Now, the angle-dependent information in surface operator $\mathbf{A}$ is clearly visible at $\tau=0$.
Note that the combination of $\mathbf{A}$ and $\mathbf{P}_{0}^{-1}$ generates all surface-related multiples in the forward data space, meaning that multiples contribute to the definition of primaries in the inverse data space. After adaptive subtraction of the surface operator around $\tau=0$ (Figure $6 \mathrm{~g}$ ), the result is transformed back into the forward data space, leading to the output without surface multiples (Figure 6h). Comparison of Figure $6 \mathrm{f}$ with $\mathrm{g}$, and Figure $6 \mathrm{~b}$ with $\mathrm{h}$ shows the large potential of multiple removal in the inverse data space, taking into account that the entire process is conceptually simple and fully data driven.

\section{CONCLUSIONS}

Seismic data is complex because of the dominant role of multiple scattering. This makes seismic processing a difficult task, requiring complex seismic tools and high-level seismic skills.

If we arrange the measurements of a seismic survey in a data matrix and we determine the inverse of this matrix, then the surface is decoupled from the subsurface. In terms of the feedback model (Figure 3), the forward path and the backward path are separated.

This decoupling property may have far-reaching consequences a)

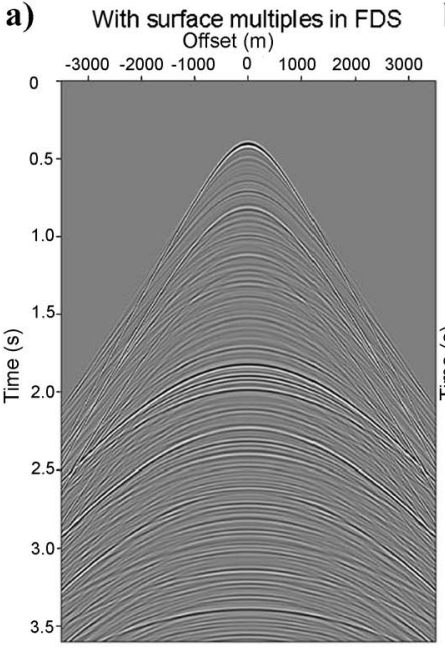

e)
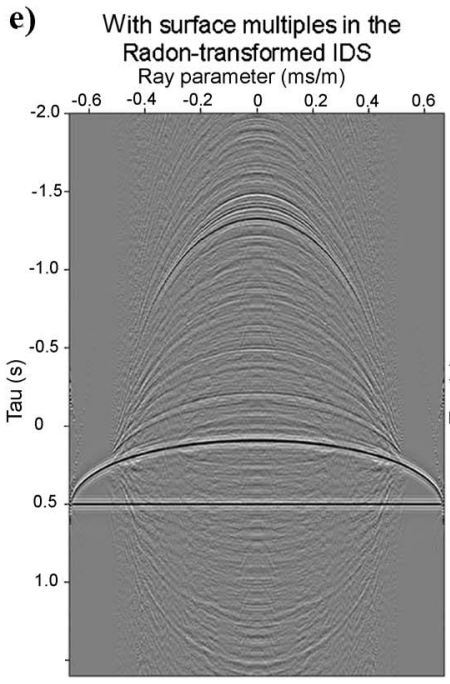

b) Without surface multiples in FDS Offset (m)
$-3000-2000-1000 \quad 0 \quad 1000 \quad 2000 \quad 3000$

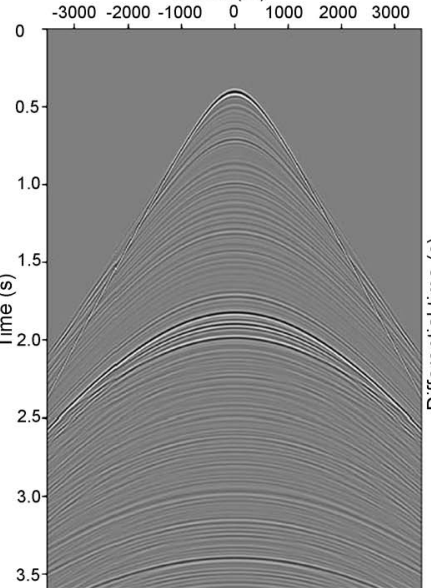

f) Without surface multiples in the

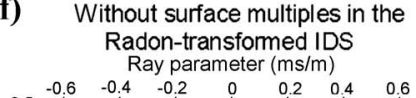

c) With surface multiples in IDS $-3000-2000-1000$ Offset (m)

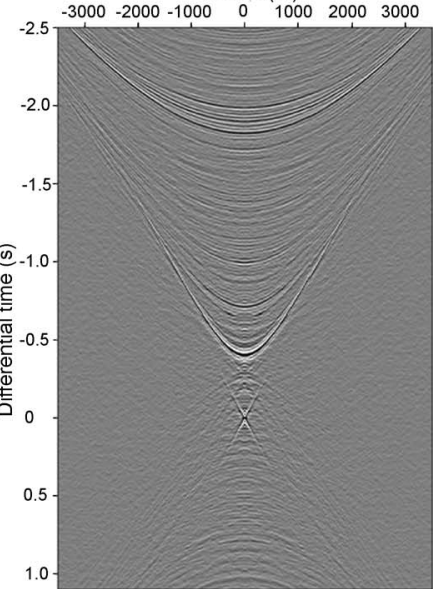

g) Removal of the surface operator

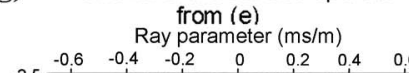

d) Without surface multiples in IDS $-3000-2000-1000 \quad 0 \quad \begin{aligned} & 0 \\ & 0\end{aligned}$

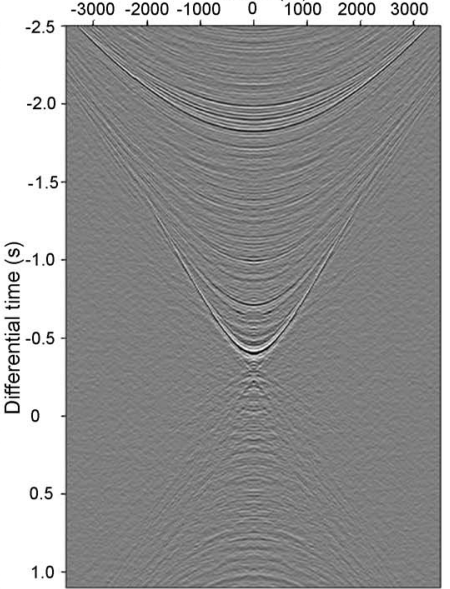

h) Transformation of $(\mathrm{g})$ back to the forward data space (FDS) Offset (m)
$-3000-2000-1000 \quad 0$
0

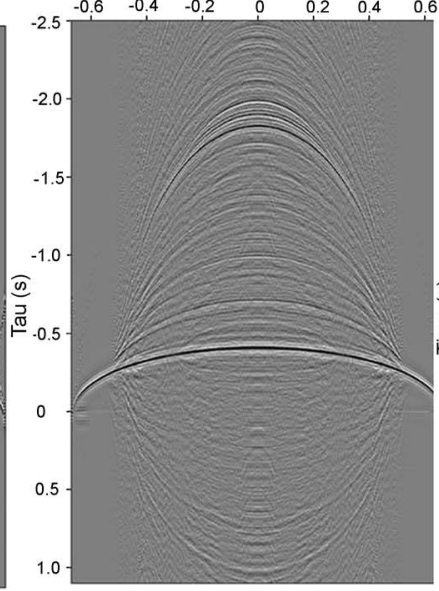

6

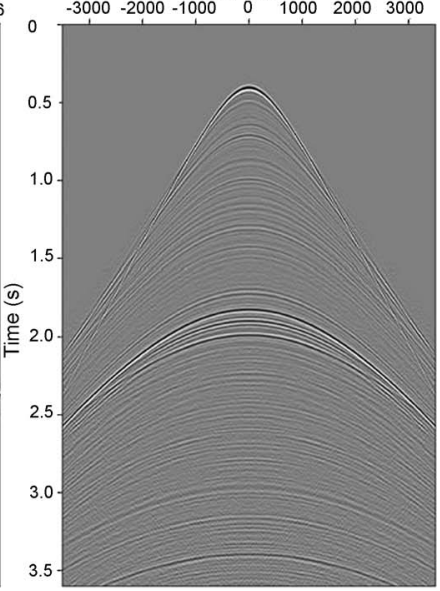

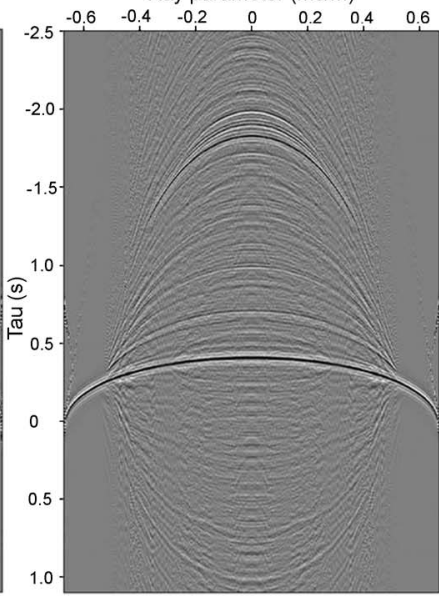

Figure 6. (a and b) Shot record modeled with and without surface-related multiples in the forward data space, (c and d) in the inverse data space, and (e and f) in the Radon-transformed inverse data space. (g) After adaptive subtraction of the surface operator around $\tau=0$, the result is transformed back to the forward data space $(h)$. 
for the way we will preprocess seismic data in the future: The angledependent scattering properties of the (near) surface as well as the angle-dependent source and detector behavior at the (near) surface can be removed from the data without prior knowledge of surface and subsurface. Moreover, it is expected that in the inverse data space, missing data can be interpolated far beyond aliasing because of the interrelationship between primaries and surface-related multiples. The latter can be understood by bearing in mind that missing angles in surface operator $\mathbf{A}$ can easily be repaired. These angles are then used in the combination of $\mathbf{A}$ and $\mathbf{P}_{0}$ to construct missing data in the forward data space.

By repeating inverse data processing at each depth level, all internal multiple scattering events related to one depth level map onto $\tau$ $=0$, and can thus be used in the imaging process. This means that by including the inverse data space, existing migration technology can be used to construct an image of the subsurface from both primaries and multiples: at each depth level, primaries are imaged in the forward data space and multiples are imaged in the inverse data space.

There are many physical systems outside the seismic discipline where feedback paths play a principal role, and where measurements can be described by integral equations of the second kind. Looking at the results of this paper, it may be expected that analysis of these systems will benefit when carried out in the inverse data space.

\section{ACKNOWLEDGMENTS}

The author would like to thank Eric Verschuur for his assistance in generating the numerical examples. The author would also like to thank the Delphi sponsors for stimulating discussions and their financial support.

\section{REFERENCES}

Berkhout, A. J., 1982, Seismic migration, imaging of acoustic energy by wave field extrapolation, Elsevier Science Publishing Company, Inc.

Kelamis, P. G., and D. J. Verschuur, 2000, Surface-related multiple elimination on land seismic data - Strategies via case studies: Geophysics, 63, 719-734.

Verschuur, D. J., 1991, Surface-related multiple elimination: an inversion approach; PhD. thesis, Delft University of Technology.

Weglein, A. B., F. A. Gasparotto, P. M. Carvalho, and R. H. Stolt, 1997, An inverse scattering series method for attenuating multiples in seismic reflection data: Geophysics, 62, 1975-1989. 